\title{
Voltage Control Areas in Transmission Systems with Distributed Generation
}

\author{
W. R. Faria ${ }^{1}$, M. E. De Oliveira ${ }^{1}$, E. S. Hoji ${ }^{2}$, G. P. Viajante ${ }^{1}$ \\ ${ }^{1}$ Federal Institute of Education, Science and Technology of Goiás at Itumbiara, NUPSE \\ Itumbiara-GO, Brazil \\ Phone/Fax number: +0055 64 21035600, e-mail: m.escobar.oliveira@gmail.com \\ ${ }^{2}$ Federal Institute of Education, Science and Technology of São Paulo at Birigui \\ Birigui-SP, Brazil.
}

\begin{abstract}
Transmission systems' main function is to transport active power from the generation source to the consumers. The presence of reactive power in a transmission system, on the other hand, is not desirable. Since the reactive power problem is characteristically local, its adjustment may be determined using devices placed near the consuming centers. Therefore, the system can be separated into regions and the reactive power problem be treated locally. This paper presents a computational tool for determining the voltage control areas (VCAs) in transmission systems based on the concept of electrical distance and the decoupled power flow method, which allows the treatment of the active and reactive power problems separately, reducing computational efforts. The presented tool also considers distributed generation and its influence on the voltage control areas. The tests have been conducted with IEEE 14 and 30 bus systems and proper conclusions are derived.
\end{abstract}

\section{Key words}

Voltage Control Areas; Distributed Generation; Reactive Power; Transmission Systems; Decoupled Power Flow

\section{Introduction}

Reactive power control is fundamental to keep constant the voltage level and vital to the safety of the electrical systems [1]. Also, transmission system capacity can be increased by minimizing the reactive power flow.

In some cases, additional reactive power sources are necessary to reach voltage and reactive power balance and set a stable operation point. The balance of voltage and reactive power can be achieved and the optimal point of operation reached. Although the correlation between voltage magnitude and reactive levels allows the existence of an optimal point, since it depends on the use of capacitors, inductors, synchronous condensers and transformers [1], finding it is an expensive and complex process.

On the other hand, it is also a known fact that, in general, the effect of voltages on power systems has a local nature, what means that the major effects of voltage variation are limited to a certain neighborhood [2] and [3]. Therefore, if the region with reactive power problems can be identified, then the voltage magnitude and reactive power can be locally controlled by implementing reactive power reserves [2].

A group of buses connected by a weak voltage boundary and presenting similar behavior to a system perturbation that may create a voltage disturbance is called a Voltage Control Area (VCA). A VCA is composed by a set of generation and load buses with voltage magnitude behavior very similar to reactive power generation and load changes outside the VCA [4] and the generators within a VCA are the primary reactive power reserve to the area [5].

The VCAs can be determined based on the concept of electrical distance between buses [2], [3], [6], [7] and [8]. Since the VCAs suggestions depends on physical characteristics such as branch conductance and susceptance, adding a small generator to the system connected near to the demand, which is the definition of distributed generation (DG) according to [9] and [10], may change the VCAs.

A computational tool for determining the VCAs in a transmission system is presented in this paper. The proposed methodology is based on the concept of electrical distance and the decoupled power flow, which leads to very similar results than the Newton-Raphson method [11], [12].

The electrical distances also allow to identify the better allocation bus for a DG to bring a load bus electrically closer to a generator and, therefore, within a VCA. Tests have been conducted with IEEE 14 and 30 bus systems and demonstrate that the VICAs allow a more accurate reactive power control.

\section{Decoupled Power Flow}

There are many mathematical ways to solve a multivariable problem, one of them being the Newton-Raphson method. This method, when applied to solving power flow, presents the following steps, as shown in [11], [13], [14]:

1- Set an initial value to bus Voltage and Angle.

2- Solve the power equations (1) and (2):

$$
\begin{aligned}
& P_{k}^{\text {calc }}=V_{k} \times\left[\sum_{m=1}^{n} V_{m} \times\left\{G_{k m} \times \cos \theta_{k m}+B_{k m} \times \sin \theta_{k m}\right\}\right] \\
& Q_{k}^{\text {calc }}=V_{k} \times\left[\sum_{m=1}^{n} V_{m} \times\left\{G_{k m} \times \cos \theta_{k m}-B_{k m} \times \sin \theta_{k m}\right\}\right]
\end{aligned}
$$


Where $\boldsymbol{G}_{\boldsymbol{k} \boldsymbol{m}}$ is the real part of admittance matrix $(\boldsymbol{Y})$ and $\boldsymbol{B}_{\boldsymbol{k m}}$ is the imaginary part of the same matrix.

3- Calculate the power mismatches:

$$
\begin{aligned}
\Delta P_{k} & =P_{k}^{\text {specified }}-P_{k}^{\text {calculated }} \\
\Delta Q_{k} & =Q_{k}^{\text {specified }}-Q_{k}^{\text {calculated }}
\end{aligned}
$$

4- Update the Jacobian Matrix:

$$
J=-\left[\begin{array}{ll}
H_{(n-1) x(n-1)} & N_{(n-1) x(n-1)} \\
M_{(n-1) x(n-1)} & L_{(n-1) x(n-1)}
\end{array}\right]
$$

Where $\mathrm{H}, \mathrm{M}, \mathrm{N}$ and $\mathrm{L}$ are matrices, obtained by (6):

$$
H=\frac{\partial P}{\partial \theta} \quad N=\frac{\partial P}{\partial V} \quad M=\frac{\partial Q}{\partial \theta} \quad L=\frac{\partial Q}{\partial V}
$$

5- Calculate the voltages and angles variations:

$$
\left[\begin{array}{l}
\Delta \boldsymbol{P} \\
\Delta \boldsymbol{Q}
\end{array}\right]^{(i)}=-J^{(i)} \times\left[\begin{array}{l}
\Delta \boldsymbol{\theta} \\
\Delta \boldsymbol{V}
\end{array}\right]^{(i)}
$$

Where $\Delta \boldsymbol{P}, \Delta \boldsymbol{Q}, \Delta \boldsymbol{\theta}$ and $\Delta \boldsymbol{V}$ are matrices.

6- Update voltages and angles

$$
\left[\begin{array}{l}
\theta \\
V
\end{array}\right]^{(i+1)}=\left[\begin{array}{l}
\theta \\
V
\end{array}\right]^{(i)}+\left[\begin{array}{l}
\Delta \theta \\
\Delta V
\end{array}\right]^{(i)}
$$

7- Return to step 2 until the convergence criteria is reached.

This method presents great accuracy when applied to Transmission Systems. There is, however, a simplification to this method which does not consider the P-V and Q- $\theta$. The Jacobian's submatrices $\mathrm{M}$ and $\mathrm{N}$ are set to zero, dropping the computational effort at the cost of loss in accuracy.

Since the power flow is just a part of the entire computational routine, the decoupled method was chosen to reduce the time to solve the problem.

\section{Mathematical Formulation of Electrical Distance}

According to [7] and [8], the transmission system can be divided in voltage control areas based on the electrical distance between buses. It is shown in [2], [3], [6], [7] and [8] that the electrical distance can be obtained from the matrix $[\partial Q / \partial V]$, which is a submatrix of Jacobian matrix by the following steps:

1- Calculate the matrix:

$$
B^{\prime \prime}=[\partial Q / \partial V]
$$

2- Invert to find the sensibility matrix:

$$
b_{i j}=\left[\partial V_{i} / \partial Q_{j}\right]
$$

Where $\mathrm{i}$ and $\mathrm{j}$ are bus numbers.

The sensibility matrix reflects the voltage reaction to a reactive power injection in a bus.

The next step is to find the voltage magnitude coupling between two buses, the matrix which shows the coupling between all buses voltages is known as attenuation matrix.
3- Attenuation matrix can be calculated by dividing each element of sensibility matrix by its diagonal value:

$$
\alpha_{i j}=b_{i j} / b_{j j}
$$

Now the electrical distance may be calculated by (12). The electrical distance represents the degree of influence caused by the voltage variation in other buses.

4- Calculate the electrical distances:

$$
D_{i j}=D_{j i}=-\log \left(\alpha_{i j} * \alpha_{j i}\right)
$$

5- Normalize the calculated distances:

$$
D_{i j}=D_{i j} / \operatorname{Max}\left(D_{i 1}, \ldots, D_{i n}\right)
$$

Calculating the electrical distance is the key to determining the VCAs using this tool and since it depends on the $[\partial Q / \partial V]$ matrix, can only be calculated after the power flow has converged.

\section{Algorithm Implementation}

\section{A. Data interpretation and matrices initialization}

The algorithm reads the data from buses and branches, then creates the fundamental matrices such as impedance, admittance, voltage magnitude, phase angle, active and reactive power (specified). In addition, a maximum error value is set which will be used as stop criteria.

\section{B. Power flow routine}

With the entry data, the algorithm performs the Decoupled Power Flow. The data is used by the next routine which suggests the voltage control areas.

\section{Suggesting voltage control areas}

The equations 9 to 13 , also presented in [8], are used to calculate the electrical distance based on the sensibility matrix, which is extracted from the power flow results.

Having the electrical distance matrix, the voltage control area suggestion is given by a hierarchical classification routine that puts generators in ranges, limited by electrical distance values.

For example, let $F_{1}, F_{2}$ and $F_{3}$ be values for electrical distance. Then the areas limits could be expressed as follows (14):

$$
\begin{aligned}
0 & \leq \text { Range } 1<F_{1} \\
F_{1} & \leq \text { Range } 2<F_{2} \\
F_{2} & \leq \text { Range } 3<F_{3}
\end{aligned}
$$

The electrical distance of each consuming bus to each generator is allocated in one of the ranges. The program suggestion is to attach a bus to the closest generator area, considering electrical distance.

The complete computational routine is represented in figure 1 as a flowchart. 


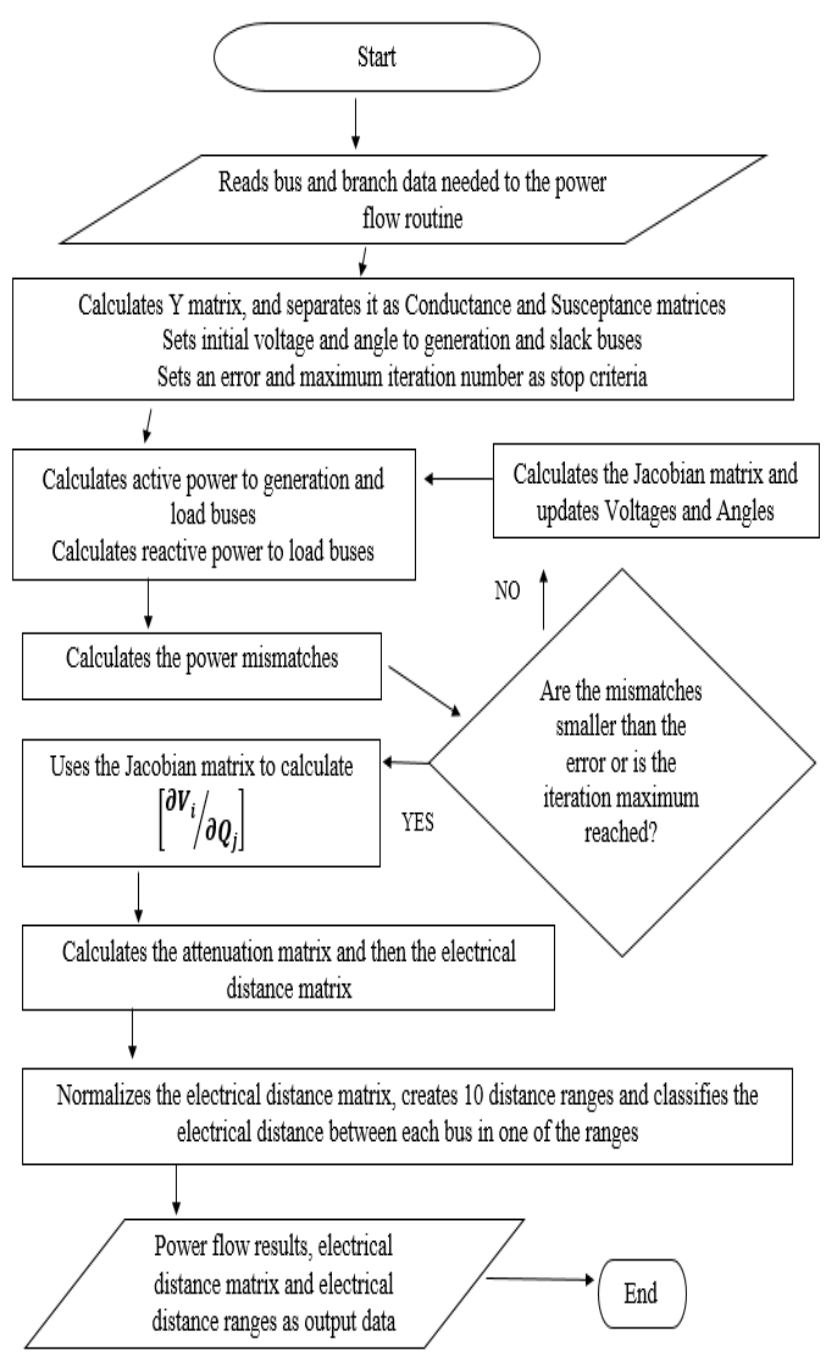

Fig. 1 - Computational tool flowchart

\section{Results}

The algorithm was tested with IEEE 14 and 30 bus systems and the results to some of the systems are shown below. The results show the electrical distance range from every load bus to each generation bus. In order to improve the VCA efficiency, the tool suggests buses with the smaller range to a common generator to be in the same VCA.

\section{A. 14 bus test system}

The original system presents 5 generation buses and 20 branches connecting the 14 buses.

Table 1 shows the electrical distance range between each bus and the generators, (one of) the smallest is highlighted to every bus. The figure 2 shows the system representation, each VCA is represented by a different color, buses on the same color are in the same VCA. In table 1 is visible that the electrical distance from any load bus to any generator is at least in range 5 .

\begin{tabular}{|c|c|c|c|c|c|c|c|c|}
\hline \multirow{2}{*}{ \#Bus } & \multirow{2}{*}{$\begin{array}{c}\text { Voltage } \\
(\mathbf{p u})\end{array}$} & \multirow{2}{*}{$\begin{array}{c}\mathbf{P} \\
(\mathbf{M W})\end{array}$} & \multirow{2}{*}{$\begin{array}{c}\mathbf{Q} \\
\text { (MVAR) }\end{array}$} & \multicolumn{5}{|c|}{ Eectrical Distance Ranges } \\
\hline & & & & 1 & 2 & 3 & 6 & 8 \\
\hline 1 & 1.060 & 23.530 & \begin{tabular}{|l}
-0.2937 \\
\end{tabular} & 1 & 10 & 10 & 10 & 10 \\
\hline 2 & 1.045 & 0.1830 & 0.0628 & 10 & 1 & 10 & 10 & 10 \\
\hline 3 & 1.010 & -0.9420 & -0.0312 & 10 & 10 & 1 & 10 & 10 \\
\hline 4 & 1.028 & -0.4780 & 0.0390 & 5 & 5 & 5 & 5 & 6 \\
\hline 5 & 1.035 & -0.0760 & -0.0160 & 5 & 5 & 5 & 5 & 6 \\
\hline 6 & 1.070 & -0.1120 & 0.4024 & 10 & 10 & 10 & 1 & 10 \\
\hline 7 & 1.046 & 0.0000 & 0.0000 & 6 & 5 & 6 & 5 & 5 \\
\hline 8 & 1.090 & 0.0000 & 0.2738 & 10 & 10 & 10 & 10 & 1 \\
\hline 9 & 1.029 & -0.2950 & \begin{tabular}{|l}
-0.1660 \\
\end{tabular} & 6 & 6 & 6 & 5 & 5 \\
\hline 10 & 1.028 & $\begin{array}{r}-0.0900 \\
\end{array}$ & -0.0580 & 6 & 6 & 6 & 5 & 5 \\
\hline 11 & 1.045 & -0.0350 & -0.0180 & 6 & 6 & 6 & 5 & 6 \\
\hline 12 & 1.053 & -0.0611 & \begin{tabular}{|c|}
-0.0161 \\
\end{tabular} & 6 & 6 & 6 & 5 & 6 \\
\hline 13 & 1.046 & \begin{tabular}{|l|}
-0.1349 \\
\end{tabular} & -0.0579 & 6 & 6 & 6 & 5 & 6 \\
\hline 14 & 1.018 & -0.1490 & -0.0500 & 6 & 6 & 6 & 5 & 6 \\
\hline
\end{tabular}

Tab. 1 - Voltage Control Areas to IEEE 14 bus test system

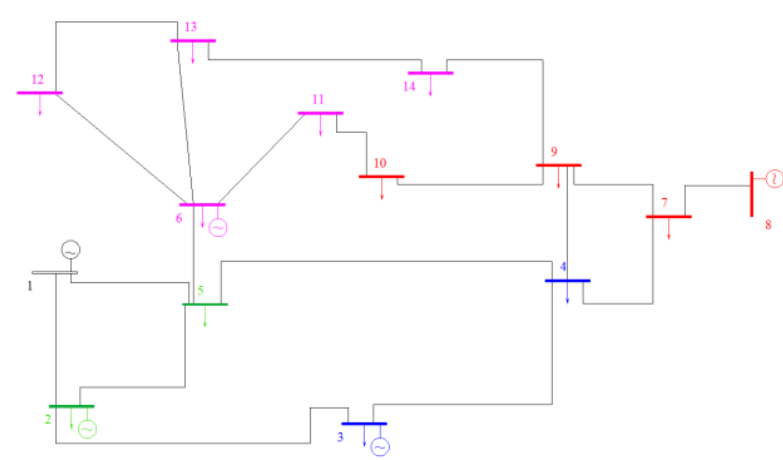

Fig. 2 - Voltage Control Areas to IEEE 14 bus test system

The bus 14 was chosen to simulate a DG. This generator represents $5 \%$ of the active generated power. That choice was made because bus 14 presents minor electrical distance to most buses, which means the VCA would be more efficient with a generator installed in bus 14 . The results to the change are shown in table 2 . The figure 2 shows the modified system' diagram.

\begin{tabular}{c|c|c|c|c|c|c|c}
\hline \multirow{2}{*}{ \#Bus } & \multirow{2}{*}{$\begin{array}{c}\text { Voltage } \\
(\mathbf{p u})\end{array}$} & \multicolumn{6}{|c}{ Eectrical Distance Ranges } \\
\cline { 3 - 8 } & $\mathbf{1}$ & $\mathbf{2}$ & $\mathbf{3}$ & $\mathbf{6}$ & $\mathbf{8}$ & $\mathbf{1 4}$ \\
\hline $\mathbf{1}$ & 1.060 & 1 & 7 & 7 & 7 & 7 & 7 \\
\hline $\mathbf{2}$ & 1.045 & 7 & 1 & 7 & 7 & 7 & 7 \\
\hline $\mathbf{3}$ & 1.010 & 7 & 7 & 1 & 7 & 7 & 7 \\
\hline $\mathbf{4}$ & 1.031 & 4 & 4 & 4 & 4 & 4 & 4 \\
\hline $\mathbf{5}$ & 1.038 & 4 & 4 & 4 & 4 & 4 & 4 \\
\hline $\mathbf{6}$ & 1.070 & 7 & 7 & 7 & 1 & 7 & 7 \\
\hline $\mathbf{7}$ & 1.049 & 4 & 4 & 4 & 4 & 4 & 4 \\
\hline $\mathbf{8}$ & 1.090 & 7 & 7 & 7 & 7 & 1 & 7 \\
\hline $\mathbf{9}$ & 1.033 & 4 & 4 & 4 & 4 & 4 & 4 \\
\hline $\mathbf{1 0}$ & 1.032 & 4 & 4 & 4 & 4 & 4 & 4 \\
\hline $\mathbf{1 1}$ & 1.047 & 4 & 4 & 4 & 4 & 4 & 4 \\
\hline $\mathbf{1 2}$ & 1.055 & 10 & 10 & 10 & 4 & 10 & 4 \\
\hline $\mathbf{1 3}$ & 1.051 & 10 & 10 & 10 & 4 & 10 & 4 \\
\hline $\mathbf{1 4}$ & 1.036 & 7 & 7 & 7 & 7 & 7 & 1 \\
\hline
\end{tabular}

Tab. $2 \longdiv { \text { -Voltage Control Areas to modified IEEE's } 1 4 \text { bus test system } }$ 


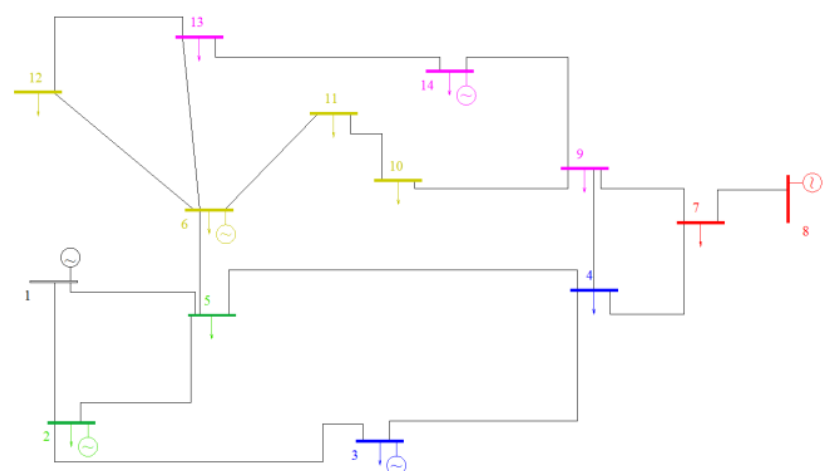

Fig. 3 - Voltage Control Areas to modified IEEE's 14 bus test system

The insertion of DG at the bus 14 directly influenced the electrical distance in most of the system buses. The changes are justified by the electrical proximity between those buses and bus 14 , which was checked on the electrical distance table, calculated by the computational tool, before the DG simulation. The changes in the ranges between buses have change to the entire system because the Jacobian matrix changes when a generation bus is added to the system. The change on the submatrix $\mathrm{L}$ affects the electrical distance matrix, hence the changes to the whole system.

\section{B. 30 bus test system}

The original system presents 6 generation buses and 41 branches connecting the 30 buses.

The suggested VCAs are shown in table 3 and the system's diagram is shown in figure 4 . The suggested VCAs to the original system has the last 3 buses fitting the last possible range to all generator buses. Using the Electrical Distance Matrix is possible to find an optimal bus to install another generator (DG) to reduce the voltage control areas range.

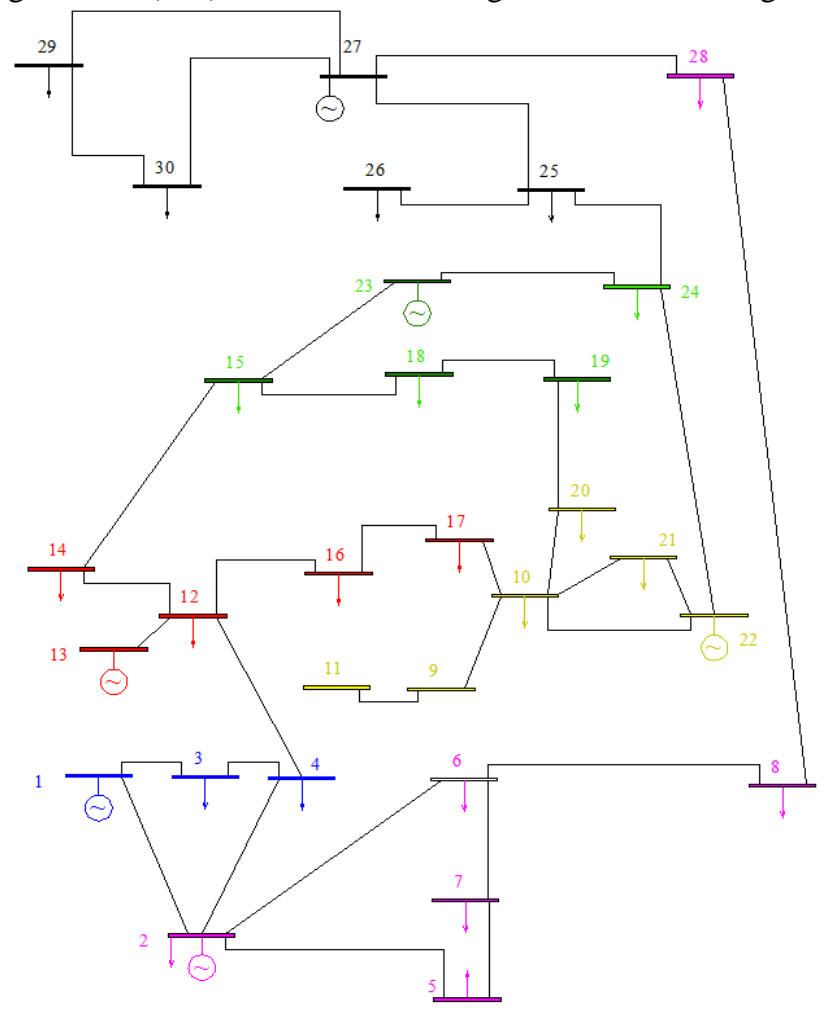

Fig. 4 - Voltage Control Areas to IEEE 30 bus test system

\begin{tabular}{c|c|c|c|c|c|c|c|c|c}
\hline \multirow{2}{*}{ \#Bus } & Voltage & $\mathbf{P}$ & $\mathbf{Q}$ & \multicolumn{6}{|c|}{ Gectrical Distance Range } \\
\cline { 5 - 9 } & (pu) & (MW) & (MVAR) & $\mathbf{1}$ & $\mathbf{2}$ & $\mathbf{1 3}$ & $\mathbf{2 2}$ & $\mathbf{2 3}$ & $\mathbf{2 7}$ \\
\hline $\mathbf{1}$ & 1.060 & 25.76 & 24.49 & 1 & 7 & 7 & 7 & 7 & 7 \\
\hline $\mathbf{2}$ & 1.043 & 39.27 & -45.56 & 7 & 1 & 7 & 7 & 7 & 7 \\
\hline $\mathbf{3}$ & 1.043 & -2.4 & -1.2 & 5 & 5 & 6 & 6 & 6 & 6 \\
\hline $\mathbf{4}$ & 1.037 & -7.6 & -1.6 & 5 & 5 & 6 & 6 & 6 & 6 \\
\hline $\mathbf{5}$ & 1.038 & 0 & 0 & 6 & 5 & 6 & 6 & 6 & 6 \\
\hline $\mathbf{6}$ & 1.029 & 0 & 0 & 6 & 5 & 6 & 5 & 6 & 6 \\
\hline $\mathbf{7}$ & 1.025 & -22.8 & -10.9 & 6 & 5 & 6 & 6 & 6 & 6 \\
\hline $\mathbf{8}$ & 1.018 & -30 & -30 & 6 & 5 & 6 & 6 & 6 & 6 \\
\hline $\mathbf{9}$ & 1.026 & 0 & 0 & 6 & 5 & 6 & 5 & 6 & 6 \\
\hline $\mathbf{1 0}$ & 1.024 & -5.8 & -2 & 6 & 5 & 6 & 5 & 6 & 6 \\
\hline $\mathbf{1 1}$ & 1.026 & 0 & 0 & 6 & 5 & 6 & 5 & 6 & 6 \\
\hline $\mathbf{1 2}$ & 1.041 & -11.2 & -7.5 & 6 & 5 & 5 & 5 & 5 & 6 \\
\hline $\mathbf{1 3}$ & 1.071 & 37 & 24.23 & 7 & 7 & 1 & 7 & 7 & 7 \\
\hline $\mathbf{1 4}$ & 1.027 & -6.2 & -1.6 & 6 & 5 & 5 & 5 & 5 & 6 \\
\hline $\mathbf{1 5}$ & 1.026 & -8.19 & -2.5 & 6 & 5 & 5 & 5 & 5 & 6 \\
\hline $\mathbf{1 6}$ & 1.025 & -3.5 & -1.8 & 6 & 5 & 5 & 5 & 6 & 6 \\
\hline $\mathbf{1 7}$ & 1.020 & -9 & -5.8 & 6 & 5 & 5 & 5 & 6 & 6 \\
\hline $\mathbf{1 8}$ & 1.013 & -3.2 & -0.9 & 6 & 6 & 5 & 5 & 5 & 6 \\
\hline $\mathbf{1 9}$ & 1.008 & -9.5 & -3.4 & 6 & 6 & 5 & 5 & 5 & 6 \\
\hline $\mathbf{2 0}$ & 1.011 & -2.2 & -0.7 & 6 & 6 & 5 & 5 & 5 & 6 \\
\hline $\mathbf{2 1}$ & 1.027 & -17.5 & -11.2 & 6 & 6 & 6 & 5 & 6 & 6 \\
\hline $\mathbf{2 2}$ & 1.033 & 21.59 & 29 & 7 & 7 & 7 & 1 & 7 & 7 \\
\hline $\mathbf{2 3}$ & 1.027 & 16 & -3.69 & 7 & 7 & 7 & 7 & 1 & 7 \\
\hline $\mathbf{2 4}$ & 1.018 & -8.7 & -6.7 & 10 & 10 & 10 & 5 & 5 & 6 \\
\hline $\mathbf{2 5}$ & 1.016 & 0 & 0 & 10 & 10 & 10 & 6 & 6 & 5 \\
\hline $\mathbf{2 6}$ & 0.9982 & -3.5 & -2.3 & 10 & 10 & 10 & 6 & 6 & 6 \\
\hline $\mathbf{2 7}$ & 1.023 & 26.91 & 1.9 & 10 & 10 & 10 & 10 & 10 & 1 \\
\hline $\mathbf{2 8}$ & 1.029 & 0 & 0 & 6 & 5 & 6 & 6 & 6 & 6 \\
\hline $\mathbf{2 9}$ & 1.003 & -2.4 & -0.9 & 10 & 10 & 10 & 10 & 10 & 5 \\
\hline $\mathbf{3 0}$ & 0.9917 & -10.6 & -1.9 & 10 & 10 & 10 & 10 & 10 & 5 \\
\hline Tab.3 - Voltage Control Areas to IEEE 30 & bus test system & &
\end{tabular}

Adding a DG to bus 25, the suggested VCAs changes to the results presented in table 4 . The modified diagram is shown in figure 5.

\begin{tabular}{c|c|c|c|c|c|c|c|c}
\hline \multirow{2}{*}{ \#Bus } & Voltage & \multicolumn{6}{|c}{ Eectrical Distance Range } \\
\cline { 3 - 8 } & $(\mathbf{p u})$ & $\mathbf{1}$ & $\mathbf{2}$ & $\mathbf{1 3}$ & $\mathbf{2 2}$ & $\mathbf{2 3}$ & $\mathbf{2 5}$ & $\mathbf{2 7}$ \\
\hline $\mathbf{1}$ & 1.060 & $\mathbf{1}$ & 4 & 5 & 5 & 5 & 9 & 7 \\
\hline $\mathbf{2}$ & 1.043 & 4 & $\mathbf{1}$ & 5 & 5 & 5 & 8 & 7 \\
\hline $\mathbf{3}$ & 1.043 & 3 & 3 & 3 & 3 & 3 & 8 & 6 \\
\hline $\mathbf{4}$ & 1.037 & 3 & 3 & 3 & 3 & 3 & 8 & 6 \\
\hline $\mathbf{5}$ & 1.038 & 3 & 3 & 3 & 3 & 3 & 8 & 6 \\
\hline $\mathbf{6}$ & 1.029 & 3 & 3 & 3 & 3 & 3 & 8 & 6 \\
\hline $\mathbf{7}$ & 1.026 & 3 & 3 & 3 & 3 & 3 & 8 & 6 \\
\hline $\mathbf{8}$ & 1.018 & 3 & 3 & 3 & 3 & 3 & 8 & 6 \\
\hline $\mathbf{9}$ & 1.026 & 3 & 3 & 3 & 3 & 3 & 8 & 6 \\
\hline $\mathbf{1 0}$ & 1.024 & 3 & 3 & 3 & 3 & 3 & 8 & 6 \\
\hline $\mathbf{1 1}$ & 1.026 & 3 & 3 & 3 & 3 & 3 & 8 & 6 \\
\hline $\mathbf{1 2}$ & 1.040 & 3 & 3 & 3 & 3 & 3 & 8 & 6 \\
\hline $\mathbf{1 3}$ & 1.071 & 5 & 5 & $\mathbf{1}$ & 5 & 5 & 9 & 7 \\
\hline $\mathbf{1 4}$ & 1.027 & 3 & 3 & 3 & 3 & 3 & 8 & 6 \\
\hline $\mathbf{1 5}$ & 1.026 & 3 & 3 & 3 & 3 & 3 & 8 & 6 \\
\hline $\mathbf{1 6}$ & 1.025 & 3 & 3 & 3 & 3 & 3 & 8 & 6 \\
\hline $\mathbf{1 7}$ & 1.019 & 3 & 3 & 3 & 3 & 3 & 8 & 6 \\
\hline $\mathbf{1 8}$ & 1.013 & 3 & 3 & 3 & 3 & 3 & 8 & 6 \\
\hline $\mathbf{1 9}$ & 1.008 & 3 & 3 & 3 & 3 & 3 & 8 & 6 \\
\hline $\mathbf{2 0}$ & 1.011 & 3 & 3 & 3 & 3 & 3 & 8 & 6 \\
\hline $\mathbf{2 1}$ & 1.027 & 3 & 3 & 3 & 3 & 3 & 8 & 6 \\
\hline $\mathbf{2 2}$ & 1.033 & 7 & 7 & 7 & $\mathbf{1}$ & 7 & 7 & 7 \\
\hline $\mathbf{2 3}$ & 1.027 & 7 & 7 & 7 & 7 & $\mathbf{1}$ & 7 & 7 \\
\hline $\mathbf{2 4}$ & 1.018 & 8 & 8 & 8 & 3 & 3 & 3 & 8 \\
\hline $\mathbf{2 5}$ & 1.017 & 10 & 10 & 10 & 7 & 7 & $\mathbf{1}$ & 7 \\
\hline $\mathbf{2 6}$ & 0.9993 & 10 & 10 & 10 & 8 & 8 & 3 & 8 \\
\hline $\mathbf{2 7}$ & 1.023 & 7 & 7 & 8 & 7 & 8 & 7 & $\mathbf{1}$ \\
\hline $\mathbf{2 8}$ & 1.030 & 3 & 3 & 3 & 3 & 3 & 8 & 6 \\
\hline $\mathbf{2 9}$ & 1.003 & 8 & 8 & 8 & 8 & 8 & 8 & 5 \\
\hline $\mathbf{3 0}$ & 0.9917 & 8 & 8 & 8 & 8 & 8 & 8 & 5 \\
\hline & & & \\
\hline
\end{tabular}

Tab. 4 -Voltage Control Areas to modified IEEE's 30 bus test System 


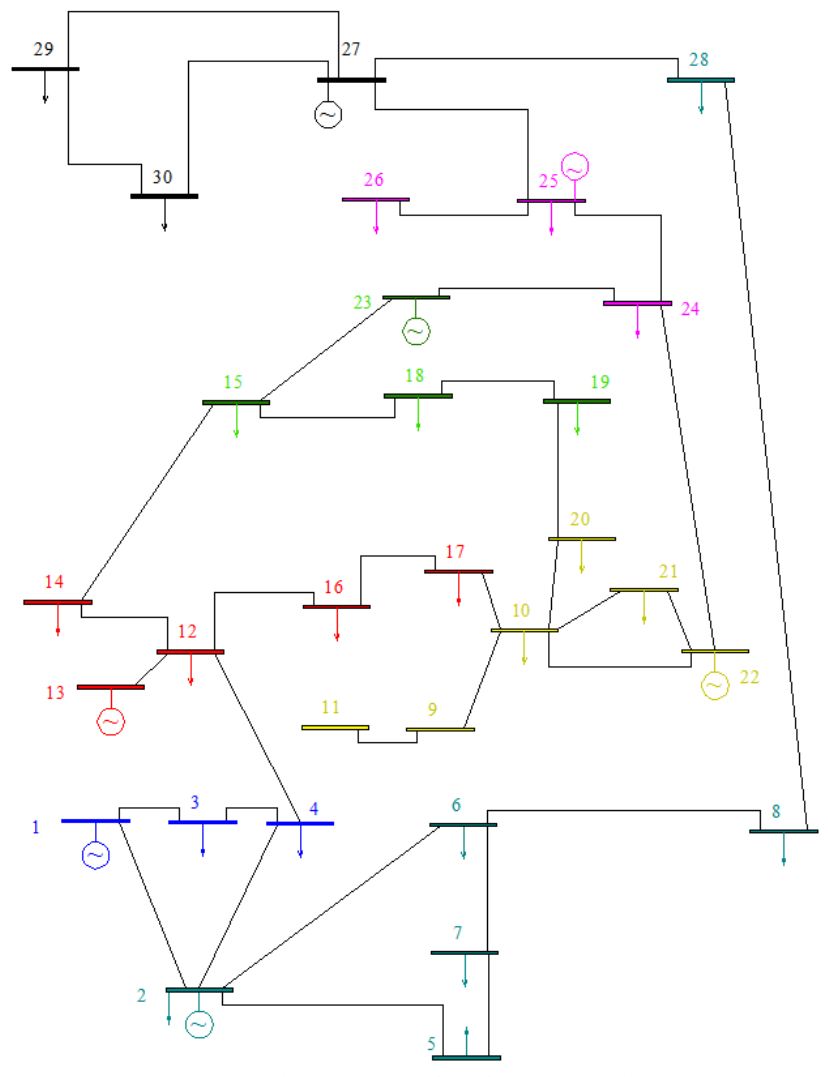

Fig. 5 - Voltage Control Areas to modified IEEE's 30 bus test system

The DG simulated on bus 25 also represents $5 \%$ of the active generated power.

Again, the choosing of bus 30 as to represent a DG was made based on its electrical distance to the buses which presented the greater range to the existing VCAs (buses 25, 26, 29 and 30). The high voltages (above 1.05pu) present on every result is owned to the input values; the Slack and Generation buses have voltage magnitude above $1.05 \mathrm{pu}$.

\section{Conclusion}

Determining the VCAs through electrical distances is a process where every load bus is grouped with the electrically closest generation bus using the sensibility matrix, which is a power flow result. The VCAs suggestions presented on this paper may be combined to the methods suggested in [7] and [8] to group VCAs attaching the ones without load buses to another VCA.

It is important to point out the use of a simple power flow method (decoupled). By doing so the program is may run online analysis enabling the user to take actions on time in critical situations. The routine can also be used for market and reactive power circulation analysis, given its power flow results.

Another important point to highlight is that connecting a DG on the right bus may change the whole VCA, even though the active power generated by it is a small portion of the total. Since the adding of a DG may improve the suggested VCAs, an algorithm which can find the optimal point to add a DG can be used as a planning tool to improve transmission systems' quality. The tool here presented provides enough data to choose an optimal point, electrically close to the excluded buses.

\section{Acknowledgement}

The authors thank the Instituto Federal de Educação, Ciência e Tecnologia de Goiás, Instituto Federal de Educação, Ciência e Tecnologia de São Paulo and NUPSE for all the support.

\section{References}

[1] T. J. E. Miller. "Reactive power control in electric systems". New York: John Wiley \& Sons Inc., 1982.

[2] H.Liu, A. Bose, V. Venkatasubramanian. "A fast voltage security assessment method using adaptive bounding". IEEE Transactions on Power Systems, vol. 14, n. 3, p.1137-1141, 2000.

[3] R. Maharjan, S. Kamalasadan. "A new approach for voltage control area identification based on reactive power sensitivities". IEEE North American Power Symposium (NAPS), 2015.

[4] R. Schlueter, I.-P. Hu, M. Chang, J. Lo, A. Costi. "Methods for determining proximity to voltage collapse" IEEE Transactions on Power Systems. vol.6, n.1, pp. 285-292, 1991.

[5] W. Villa, J. Rueda, S. Torres, W. Peralta. "Identification of voltage control areas in power systems with large scale wind power integration". Transmission and Distribution: Latin America Conference and Exposition, pp.1-7, 2012.

[6] E. Nobile, A. Bose. "AAA new scheme for voltage control in a competitive ancillary service market". Proceedings of PSCC'02, $14^{\text {th }}$ Power System Computation Conference, 2002.

[7] P. Lagonotte, J.C. Sabonnadiere, J.-Y. Leost. "Structural analysis of the electrical system: application to secondary voltage control in france". IEEE Transactions on Power Systems, vol.4, n.2, p.479486, 1989.

[8] J. Zhong, E. Nobile, A. Bose. "Localized reactive power markets using the concept of voltage control areas". IEEE transactions on power systems, New York, v.19, n.3, p.1555-1561, 2004.

[9] K. Divya, S. Srinivasan. "Optima Siting and Sizing of DG in Radial Distribution System and Identifying Fault Location in Distribution System Integrated with Distributed Generation". International Conference on Advanced Computing and Communication Systems, Coimbatore, 2016

[10] J. J. Grainger, W. D. Stevenson, Power System Analysis. New York: McGraw-Hill, 1994.

[11] H. Mohamad, Z. Zakaria, M. Z. B. Mazlan. "Development of GUI Power System Load Flow Analysis Tool based on Newton Raphson Method". IEEE $7 \mathrm{~h}$ International Conference on Enineering Education, 2015.

[12] X. S. Zhang, Z. L., E. K. Yu, J. Ch. Chen, "A comparison on power flow calculation methods for distribution network". Power System Technology, vol.22, p.45-49, 1998.

[13] R. J. Kaye, F. F. WU. "Analysis of Linearized Decoupled Power Flow Approximations for Steady-State Security Assessment". IEEE Transactions on circuits and systems. vol. 31, n.7, 1984.

[14] M. Tolikas, L. Trajkovic, M. D. Ilic. "Homotopy Methods for Solving Decoupled Power Flow Equations". IEEE International Symposium on Circuits and Systems. vol.6, p.2833-2839, 1992. 